\title{
Correlative Live-Cell, Electron Microscopy and Nanoscale Secondary Ion Mass Spectrometry Elucidates the Mechanism for the Release of Cholesterol-Rich Particles from the Plasma Membrane of Macrophages
}

Thomas A. Weston ${ }^{1 *}$, Xuchen $\mathrm{Hu}^{1}$, Cuiwen $\mathrm{He}^{1}$, Rachel S. Jung ${ }^{1}$, Loren G. Fong ${ }^{1}$, Haibo Jiang ${ }^{2}$ and Stephen G. Young ${ }^{1}$

${ }^{1}$ University of California Los Angeles, Department of Medicine. Los Angeles, United States of America

${ }^{2}$ University of Western Australia, School of Biomedical Sciences. Perth, Australia

* Corresponding Author: tweston@mednet.ucla.edu

Macrophages move actively throughout the body to locate and ingest dead cells, clean up cellular debris and foreign materials, and take up excess lipoproteins. As a result, macrophages can become overloaded with cholesterol, which can lead to the formation of foam cells that are associated with the development of atherosclerosis [1]. Macrophages have been thought to unload surplus cholesterol through direct interactions between $\mathrm{ABC}$ transporters on the plasma membrane and cholesterol acceptors in the plasma (e.g., HDL) [2]. However, recent studies in our group and others have shown that macrophages also release 20- to 120-nm particles enriched in cholesterol.[3-7]. Thus far, the biogenesis of these cholesterol-rich particles has remained mysterious and techniques to visualize cholesterol efflux have been limited. We suspected that a combination of live-cell time-lapse imaging, electron microscopy, and nanoscale secondary ion mass spectrometry (NanoSIMS) would make it possible to define the formation of macrophage particles. In the current study, we observed that macrophages release enormous numbers of particles from the plasma membrane during movement of lamellipodia and filopodia. These particles remain attached to the substrate in close proximity to the macrophages. NanoSIMS imaging revealed that the "lawn" of particles on the substrate surrounding macrophages is highly enriched in "accessible cholesterol".

In our first experiments, macrophages were plated onto poly-D-lysine-coated silicon wafers and release of particles was visualized by SEM. SEM imaging revealed a "lawn" of 20- to 120-nm particles on the substrate surrounding the macrophage. The particles were mostly seen polarized to one or two sides of the cell (Fig. 1). This led us to believe that these particles are left behind on the substrate during cellular movement. To test this assertion, we performed live-cell time-lapse microscopy in order to track the movement of the cells, then correlated these movies with SEM and NanoSIMS.

Macrophages were plated on poly-D-lysine-coated gridded Mattek ${ }^{\mathrm{TM}}$ cell culture dishes that were precoated with $\sim 3-\mathrm{nm}$ of iridium. Cells were imaged by live-cell time-lapse microscopy for $24 \mathrm{~h}$, at $5 \mathrm{~min}$ intervals. The next day, cells were incubated for $2 \mathrm{~h}$ at $4{ }^{\circ} \mathrm{C}$ with $\left[{ }^{15} \mathrm{~N}\right] \mathrm{ALO}-\mathrm{D} 4$, a modified cytolysin that binds to accessible cholesterol. Cells were then fixed and air dried. The glass coverslips were removed from the bottom of the cell culture dishes, mounted onto aluminum SEM stubs, and coated again with 3$\mathrm{nm}$ of iridium. Samples were imaged with SEM followed by NanoSIMS. Correlative images were taken of the same field as live-cell images.

Live-cell imaging and SEM revealed that the release of large "lawns" of 20- to 120-nm particles was associated with movement of lamellipodia and filopodia (Fig 2A-B). NanoSIMS imaging revealed that the particles were highly enriched in accessible cholesterol (Fig. 2C-D). We believe that the release of accessible cholesterol-rich particles from the macrophage plasma membrane occurs during cellular 
movement, more specifically the adhesion and release of lamellipodia and filopodia. Correlative live-cell, electron microscopy, and NanoSIMS (CLEMNS) was imperative for our studies especially due to the ability to resolve an isotopic tag ([ $\left.\left.{ }^{15} \mathrm{~N}\right] \mathrm{ALO}-\mathrm{D} 4\right)$ at high resolution provided by the NanoSIMS. We are certain that this technique will be applicable in a wide range of experiments as it can be modified with isotopic labeling of different cell types, proteins, nucleotides, antibodies or possibly drugs.

\section{References:}

[1] XH Yu et al., Clinica chimica acta; international journal of clinical chemistry 424 (2013), p. 245.

[2] PT Duong et al., J Lipid Res 47 (2006), p. 832.

[3] AR Tall, P Costet and N Wang, J Clin Invest 110 (2002), p. 899.

[4] C He et al., Proc Natl Acad Sci U S A 115 (2018), p. E8499.

[5] SR Freeman et al., J Lipid Res 55 (2014), p. 115.

[6] A Hafiane and J Genest, Atherosclerosis 257 (2017), p. 90.

[7] X Jin et al., Arterioscler Thromb Vasc Biol 38 (2018), p. 1504.

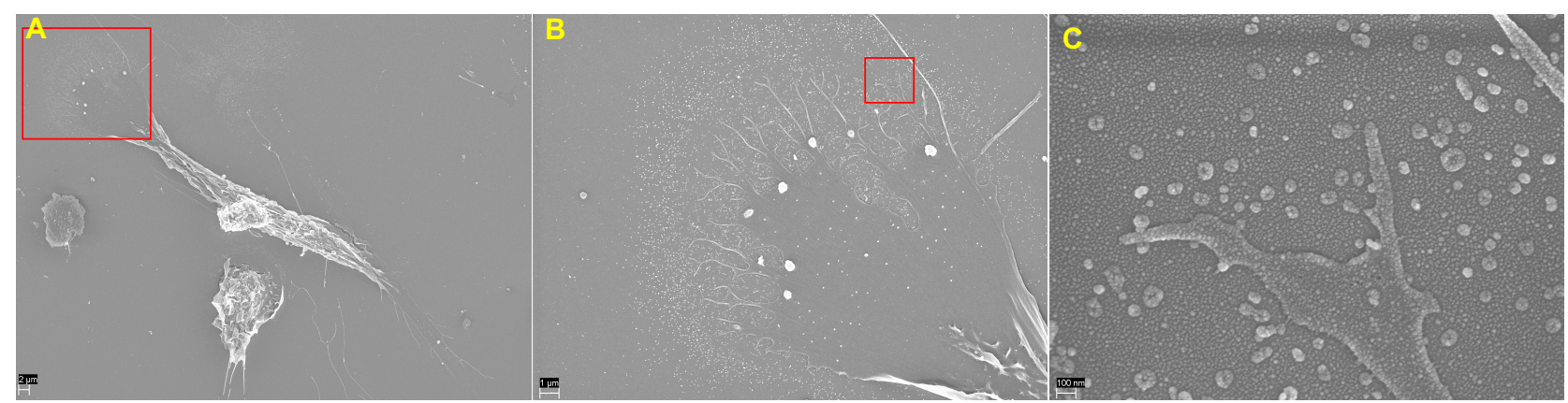

Figure 1. Particles Released by Macrophages Appear in Close Proximity to the Cells and are Formed at the Cell-Substrate Interface. A. SEM image showing a macrophage with particles polarized to one end. B. A lawn of particles in close proximity to the filopodia. C. Particles forming at the cellsubstrate interface.

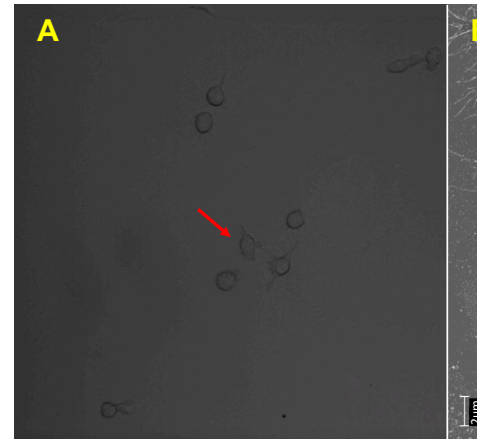

Live-Cell

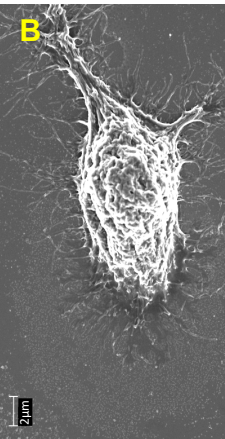

SEM

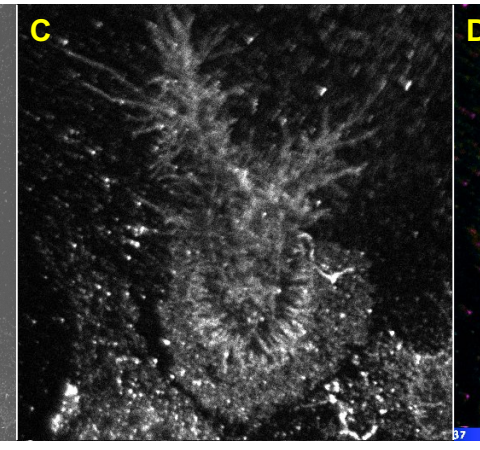

${ }^{14} \mathrm{~N}$

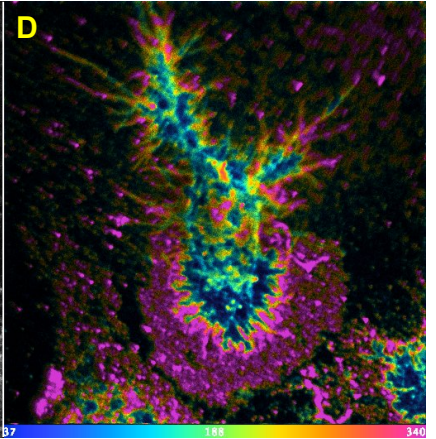

${ }^{15} \mathrm{~N} /{ }^{14} \mathrm{~N}$

Figure 2. Correlation of Live-Cell, Scanning Electron Microscopy, and NanoSIMS A. Final frame of live-cell time-lapse microscopy video showing a cell with high filopodial movement. Field-width: $320 \mu \mathrm{m}$ B. Correlative scanning electron micrograph showing a lawn of particles in close proximity to filopodia. C. ${ }^{14} \mathrm{~N}$ NanoSIMS image shows cell morphology Field-width: $40 \mu \mathrm{m}$ D. ${ }^{15} \mathrm{~N} /{ }^{14} \mathrm{~N}$ NanoSIMS image showing enrichment of ${ }^{15} \mathrm{~N}$ in the particles and the filopodia compared to the cell body. Field-width: $40 \mu \mathrm{m}$. 